International Conference Mathematical and Computational Biology 2011

International Journal of Modern Physics: Conference Series

Vol. 9 (2012) 11-23

(C) World Scientific Publishing Company

DOI: $10.1142 / \mathrm{S} 2010194512005053$

\title{
A MATHEMATICAL APPROACH TO STRUCTURAL TRANSITIONS IN VIRAL CAPSIDS
}

\author{
GIULIANA INDELICATO \\ Centre for Complex Systems Analysis, University of York, Heslington, York, UK \\ giuliana.indelicato@york.ac.uk \\ REIDUN TWAROCK \\ Centre for Complex Systems Analysis, University of York, Heslington, York, UK \\ reidun.twarock@york.ac.uk
}

\begin{abstract}
The majority of viruses have protein containers, called capsids, in which proteins are arranged with icosahedral symmetry. The capsids of these viruses follow structural blueprints that can be modelled as subsets of 3-dimensional aperiodic lattices called quasicrystals. These, in turn, can be obained by projection from 6-dimensional Bravais lattices. In this work we apply the crystallographic theory of phase transitions to these $6 \mathrm{D}$ lattices, and via projection of these results derive information on the structural transitions of viruses important for infection.
\end{abstract}

Keywords: Virus structure; structural transitions in viral capsids; Bain strain; phase transitions in quasicrystals.

\section{Introduction}

Viruses are examples of order at the nanoscale. In their simplest form, viruses consist of containers formed from protein, into which the genomic material is packaged. Crick and Watson suggested that these viral containers are organised according to symmetry for reasons of genetic economy. ${ }^{9}$ Symmetry implies that multiple copies of the same protein are used for the construction of the containers. Such containers can therefore be coded by relatively short genomic sequences, that can be more easily accommodated in the volume of the container. Caspar and Klug explained that larger viruses follow an additional rule set (quasi-equivalence) that provides constraints on how viruses organise their protein subunits in areas that are not constrained by symmetry (i.e. in the fundamental domain of the symmetry group, also called asymmetric unit in biology). ${ }^{7}$ Their approach resulted in a classification of viral capsid structures in terms of triangulations. It has been incorporated into a larger framework, called Viral Tiling theory, that also accomodates non-quasiequivalent cases, such as the cancer-causing Polyomaviridae, by generalising these triangulations to tilings in terms of different shapes of building blocks (tiles). ${ }^{17,31,32}$ 
These approaches all predict the locations of the proteins in the viral capsids, but by construction do not provide any information on the sizes and structures of the individual capsid proteins, or, on the structural relation between the protein container and the packaged genomic material. In order to obtain such information, an affine extension of the symmetry group is necessary, that relates different radial levels of a structure, exhibiting icosahedral symmetry overall, with each other. A classification of affine extensions of the icosahedral group has been derived by Keef et al. ${ }^{18}$ and the actions of the affine extended symmetry groups have been encoded by point arrays. The classification of affine extensions of the icosahedral group has resulted in a library of such point arrays, and has been demonstrated that this library provides unprecedented insights into virus geometry. ${ }^{15}$

We use this library here as a starting point for our analysis of the structural transitions in viruses. The capsids of many viruses must undergo structural rearrangements for these particles to become infective. Examples include the maturation of the viral capsid from a procapsid into an expanded form in HK97, or the opening of pores in CCMV, TBSV or RCNMV, through which the genomic material is ejected. ${ }^{24,26,27}$ Following Indelicato et al. ${ }^{11}$ we demonstrate that such structural transitions can be understood via a crystallographic approach as illustrated in Fig. 1. In particular, we represent the virus before and after expansion

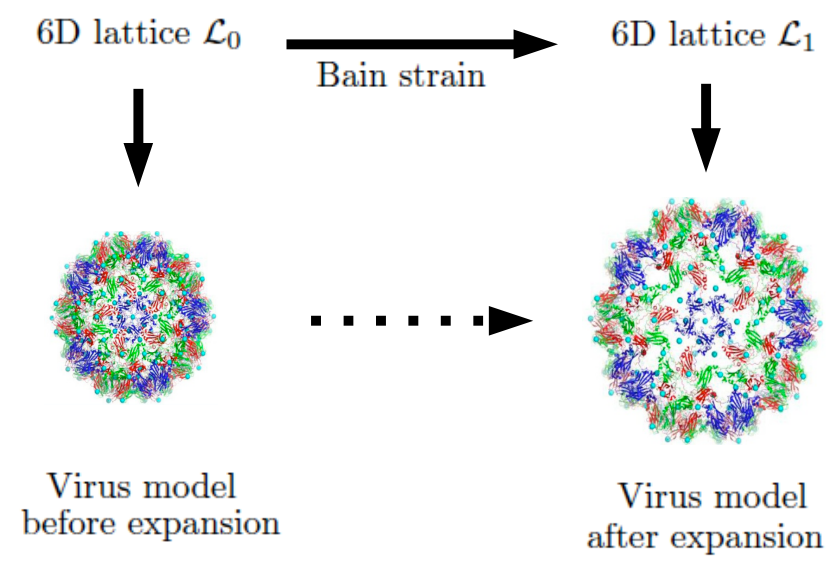

Fig. 1. Sketch of the procedure: we use 6D Bain-like transitions to predict conformational changes of models of $3 \mathrm{D}$ viral capsids.

by the appropriate element in the library of point arrays, and associate with these point arrays lattices in $6 \mathrm{D}$ such that all points of the array can be obtained from lattice vectors via projection. We then analyse lattice transitions between these $6 \mathrm{D}$ lattices via a crystallographic approach akin to the one used in chemistry and physics for the classification of lattice transitions. ${ }^{1-4,6,8,13,20,22,23,29,35}$ An example of a Bain transformation in $3 \mathrm{D}$ is shown in Fig. 2. It is a transition from an FCC 

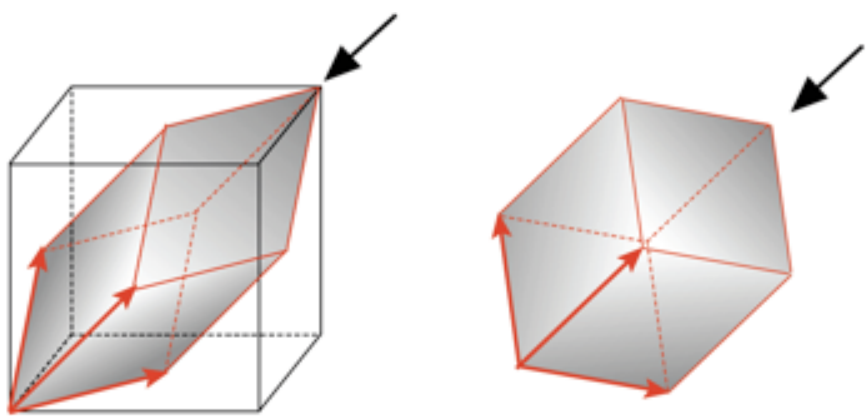

Fig. 2. An example of a Bain transition in 3D. The basis vectors corresponding to a FCC lattice (left) are deformed along a rhombohedral pathway into those of a SC lattice. The transition is distinguished by the fact that it can be realised by varying a single parameter, i.e., the angle between the basis vectors.

(left) to a SC (right) lattice that is governed by variation of a single parameter, corresponding to the angle between neighbouring basis vectors. Transformations that require variation of a minimal number of parameters (Bain transformations) are of particular interest in chemistry and physics. Similarly, we are seeking here Bain transformations in 6D, and then infer, via projection, information on the transition paths between the point array of the start and end configuration of the virus. In this way, we obtain structural boundary conditions on the organisation of viral capsids during transition.

In Section 2 we discuss the discrete approximation of capsid geometry via the library of point arrays, and illustrate these geometric constraints for the case of CCMV both before and after expansion. We moreover show how these constraints can be embedded into a $6 \mathrm{D}$ lattice picture. In Section 3 we define the mathematical concepts needed to understand capsid transitions, and show how 6D lattice transitions induce capsid transitions in 3D. Applications to CCMV are discussed in detail in Section 4. We conclude with an evaluation of this approach.

\section{Discrete Approximations of Capsid Geometry}

The structures of icosahedral viral capsids can be encoded by $3 \mathrm{D}$ point arrays corresponding to the classification of affine extensions of the icosahedral group in Keef et $a l .{ }^{18}$ The library of point arrays resulting from a classification of affine extensions of the icosahedral group has a simple description in terms of superpositions of copies of two nested polyhedra translated along suitable vectors. Consider a 'skeletal' double-shell structure, given as the union of two nested polyhedra (either an icosahedron, a dodecahedron or an icosidodecahedron) with specific relative radii corresponding to the point arrays in the library. ${ }^{18}$ The full point array is obtained via translation of the double-shell structure by one of a finite set of values listed in this reference, along the directions of a common symmetry axis of the polyhedra 
forming the double-shell. The classification shows that there are in total 342 such combinations.

We define the standard icosahedron of length $\sqrt{2+\tau}$, denoted by $I C O$, as the icosahedral orbit of the vector $(\tau, 1,0)$ in $\mathbb{R}^{3}$; the standard icosidodecahedron of length $\tau$, denoted by $I D D$, as the icosahedral orbit of $\left(\tau, \tau^{2}, 1\right)$, and the standard dodecahedron of length $\sqrt{3}$, denoted by $D O D$, as the icosahedral orbit of $(1,1,1)$. We call the union of two rescaled standard polyhedra, each of the form $2^{h} \tau^{k} I C O$, $2^{h} \tau^{k} I D D$ or $2^{h} \tau^{k} D O D$, with $k \in \mathbb{Z}$ and $h \in\{-1,0,1\}$, a (viral) skeletal configuration as it completely determines our discrete model. Each skeletal configuration is completely defined, modulo a global rescaling, by two numbers (the pair $x-y$ ), that indicate two rescaled standard polyhedra (cf. Table 3 for the list of skeletal configurations relevant to the description of CCMV). Each number $x$ uniquely identifies a pair composed of a standard polyhedron and a translation vector along a symmetry axis of the icosahedral group, together with a scaling factor that indicates its length relative to the size of the standard polyhedron (cf. Table 2). For instance, the skeletal configuration labeled by $10-44$ is the union of an icosahedron (structure 10, translation vector $\tau^{2} D O D$ ), and an icosidodecahedron rescaled by $2 \tau$ (structure 44 , translation vector $\left.\frac{1}{2} \tau D O D\right)$. Note that rescaling by the factor $2 \tau$ transforms the translation vector of the structure 44 into the translation vector of the structure 10. Therefore, a skeletal configuration with label $x-y$ uniquely identifies both the relative sizes of the two standard polyhedra and a common translation vector $\boldsymbol{s}_{x-y}$.

A point array, or viral configuration, $S$ is the point set obtained by adding to a skeletal configuration with label $x-y$, its translates by the icosahedral orbit of the vector $\boldsymbol{s}_{x-y}$.

Let $\mathcal{I}_{3}$ denote a $3 \mathrm{D}$ representation of the icosahedral group $\mathcal{I}$. If $\boldsymbol{u}, \boldsymbol{r}, \boldsymbol{s}$ are vectors pointing along a 2,3 , or 5 - fold axis of $\mathcal{I}_{3}$, then the icosahedral orbits $\mathcal{I}_{3} \boldsymbol{u}$, $\mathcal{I}_{3} \boldsymbol{r}$ and $\mathcal{I}_{3} s$ correspond to the standard polyhedra defined above. For every element $S$ in the classification of icosahedral point arrays, ${ }^{18}$ there exists $\boldsymbol{u}, \boldsymbol{r}$ and $\boldsymbol{s}$ as above such that

$$
S \equiv S(\boldsymbol{u}, \boldsymbol{r}, \boldsymbol{s})=\mathcal{I}_{3} \boldsymbol{u} \cup \mathcal{I}_{3} \boldsymbol{r} \cup\left(\mathcal{I}_{3} \boldsymbol{u}+\mathcal{I}_{3} \boldsymbol{s}\right) \cup\left(\mathcal{I}_{3} \boldsymbol{r}+\mathcal{I}_{3} \boldsymbol{s}\right)
$$

Experimental data concerning the structures of viral capsids are available as pdbfiles from either the Protein data Bank or the ViPER website. ${ }^{33}$ Via the algorithm in Keef et al. ${ }^{15}$ it is possible to associate with the pdb-file of any given virus the appropriate viral configuration that best encodes the features of its geometry. For CCMV, the point arrays have been determined based on the pdb-files with ID 1cwp for the pre-, and ccmv_swln_1 for the post-transition configuration. ${ }^{34}$ Starting from the pdb data, the algorithm first locates the outermost features of the virus, and rescales and aligns the icosahedral point sets so that the external points of the set are as close as possible to them. Then, a root mean square deviation analysis (RMSD) is performed to score how well the point sets fit around the proteins of the capsid. A second score (topology score) is calculated, measuring how well the sets of points encode the features of the external surface of the capsid. The final ranking 
of the points set results from a combination of the RMSD and the topology scores (see also $^{16}$ for examples).

The points of these arrays are located near structurally or functionally important geometric features of the capsid. ${ }^{15}$ For an example, see the match of points with the capsid of Cowpea Chlorotic Mottle virus (CCMV) before and after expansion in Fig. 3. The points around a hexameric cluster of proteins in the capsid are shown in magnification.

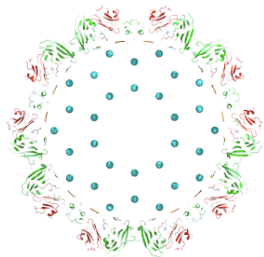

(a)

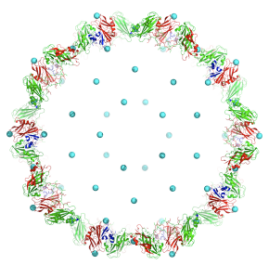

(d)

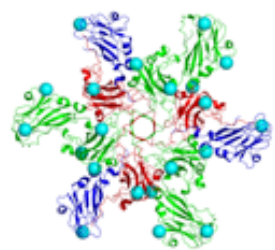

(b)

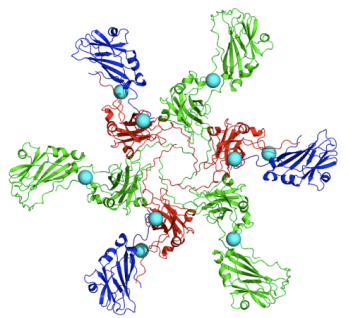

$(e)$

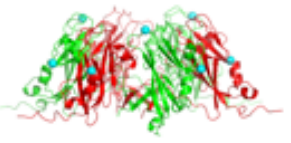

$(c)$

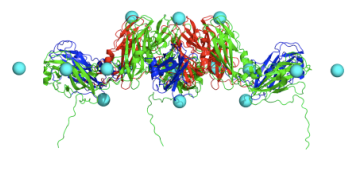

$(f)$

Fig. 3. A 3D point array modelling the layout of a viral capsid before expansion (top line), after expansion (bottom line). The approximant point array (blue dots) is shown superimposed on (a, d) a section of the viral capsid, (b, e) a top view, and (c, f) a side view, of a of a cluster of six proteins (hexamer).

\section{1. $6 D$ icosahedral lattices}

Since we are going to relate point arrays with $6 \mathrm{D}$ lattices, we start by introducing lattices with icosahedral symmetry in $6 \mathrm{D}$.

Given a basis $\left\{\boldsymbol{b}_{\alpha}\right\}_{\alpha=1, \ldots, 6}$ in $\mathbb{R}^{6}$, we indicate by $B \in G L(6, \mathbb{R})$ the matrix with columns given by the components of the vectors $\boldsymbol{b}_{\alpha}$ in the canonical basis $\left\{\boldsymbol{e}_{\alpha}\right\}_{\alpha=1, \ldots, 6}$ of $\mathbb{R}^{6}$. We write

$$
\mathcal{L}(B)=\left\{\boldsymbol{x}=\sum_{\alpha=1}^{6} m^{\alpha} \boldsymbol{b}_{\alpha}: m^{\alpha} \in \mathbb{Z}\right\}
$$


for the Bravais lattice with basis $B$. Note that every other lattice bases have the form $B M$, with $M \in G L(6, \mathbb{Z})$. Throughout this paper we assume that all bases have the same orientation in $\mathbb{R}^{6}$.

The symmetry of the lattice is encoded by the lattice group of $\mathcal{L}(B)$ is

$$
\Lambda(B)=\{M \in G L(6, \mathbb{Z}): \exists Q \in S O(6) \text { such that } Q B=B M\},
$$

and the point group

$$
\mathcal{P}(B)=\{Q \in S O(6): \exists M \in G L(6, \mathbb{Z}) \text { such that } Q B=B M\} .
$$

In what follows we will focus on the simple cubic (SC), body-centered cubic (BCC) and face-centered cubic (FCC) $6 \mathrm{D}$ lattices, given by: ${ }^{19}$

$$
\begin{aligned}
& \mathcal{L}_{S C}=\left\{\boldsymbol{x}=\left(x_{1}, \ldots, x_{6}\right): x_{i} \in \mathbb{Z}, i=1, \ldots, 6\right\}, \\
& \mathcal{L}_{B C C}=\left\{\boldsymbol{x}=\frac{1}{2}\left(x_{1}, \ldots, x_{6}\right): x_{i} \in \mathbb{Z}, x_{i}=x_{j} \bmod 2, i, j=1, \ldots, 6\right\}, \\
& \mathcal{L}_{F C C}=\left\{\boldsymbol{x}=\frac{1}{2}\left(x_{1}, \ldots, x_{6}\right): x_{i} \in \mathbb{Z}, \sum x_{j}=0 \bmod 2\right\} .
\end{aligned}
$$

The common point group $\mathcal{P}_{C}$ of the cubic lattices (of $\left(6 ! 2^{5}\right)$-elements) is $\mathcal{P}_{C}=$ $S O(6) \cap G L(6, \mathbb{Z})$. The lattice groups of the three cubic lattices do not coincide. Indeed, they are not even conjugate in $G L(6, \mathbb{Z})$ (this is the defining property for lattices with distinct Bravais types ${ }^{23}$ ).

Let $\mathcal{I}$ be a $6 \mathrm{D}$ representation of the icosahedral group (cf. e.g., $\mathrm{Katz}^{12}$ ): the matrix group $\mathcal{I}$ leaves all three $6 \mathrm{D}$ cubic lattices (SC, FCC, BCC) invariant, and is a subgroup of their common cubic point group in the standard basis. As the three $6 \mathrm{D}$ cubic lattices are the only $6 \mathrm{D}$ lattices with this property, ${ }^{19}$ they are also often referred to as the 'icosahedral' $6 \mathrm{D}$ lattices.

The action of $\mathcal{I}$ on $\mathbb{R}^{6}$ decomposes into two non-equivalent irreducible representations of dimension $3 .{ }^{12}$ Therefore, the $6 \mathrm{D}$ space splits into the direct sum of two 3D subspaces which are invariant under icosahedral symmetry and are orthogonal to each other: the parallel space, and the orthogonal space, denoted by $E^{\|}$and $E^{\perp}$, respectively, and we write $\pi: \mathbb{R}^{6} \rightarrow E^{\|}$and $\pi^{\perp}: \mathbb{R}^{6} \rightarrow E^{\perp}$ for the corresponding projections. In particular, ${ }^{12}$ we choose as parallel space $E^{\|}$the subspace in which the orthogonal projection of the standard basis $\left(\boldsymbol{e}_{\alpha}\right)_{\alpha=1, \ldots, 6}$ corresponds to the vectors pointing to the vertices of an icosahedron.

We denote by $\mathcal{I}_{3}$ the $3 \mathrm{D}$ representation of $\mathcal{I}$ on $E^{\|} . Q^{\prime} \in \mathcal{I}_{3}$ corresponds to $Q \in \mathcal{I}$ in this representation if

$$
Q^{\prime} \pi \boldsymbol{v}=\pi Q \boldsymbol{v}
$$

for all $\boldsymbol{v} \in \mathbb{R}^{6}$. Hence, icosahedral orbits in $\mathbb{R}^{6}$ project to icosahedral orbits in $\mathbb{R}^{3}=E^{\|}$. The same property holds for any subgroup $\mathcal{G} \subset \mathcal{I}: \mathcal{G}$-orbits in $\mathbb{R}^{6}$ project to $\mathcal{G}_{3}$-orbits in $\mathbb{R}^{3}$, where $\mathcal{G}_{3}$ is the $3 \mathrm{D}$ representation of $\mathcal{G}$ in $E^{\|}$.

In addition to the point group operations, an important symmetry operation of the cubic lattices in $6 \mathrm{D}$ is the quasi-dilatation $D$ (cf. ${ }^{11}$ ): the BCC and FCC lattices are invariant under the action of $D$, and the SC lattice is invariant under $D^{3}$. The 
transformation $D$ acts as a dilatation by a factor of $\tau$ in $E^{\|}$and by $1 / \tau$ in $E^{\perp}$, where $\tau=\frac{1}{2}(1+\sqrt{5})$.

\subsection{Embedding of capsid geometry into a $6 D$ icosahedral lattice via the cut-and-project method}

In order to associate a point array with a $6 \mathrm{D}$ lattice, we use the following facts:

(i) The projection $\pi$ is one-to-one onto its image when restricted to $\mathcal{L}$, since $E^{\|}$is totally irrational (i.e., $E^{\|} \cap \mathcal{L}=\{0\}$ ) with respect to $\mathcal{L}_{S C}, \mathcal{L}_{F C C}$ and $\mathcal{L}_{B C C}$.

Table $1.6 \mathrm{D}$ vectors and their $3 \mathrm{D}$ projections corresponding to the vertices of the standard polyhedra. Vectors are listed modulo inversion.

\begin{tabular}{|c|c|c|}
\hline & Orbits in 6D modulo inversion & $3 \mathrm{D}$ representation \\
\hline Icosahedron & $\begin{array}{l}(1,0,0,0,0,0) \\
(0,1,0,0,0,0) \\
(0,0,1,0,0,0) \\
(0,0,0,1,0,0) \\
(0,0,0,0,1,0) \\
(0,0,0,0,0,1)\end{array}$ & $\begin{array}{l}(\tau, 1,0) \\
(0, \tau, 1) \\
(-1,0, \tau) \\
(0,-\tau, 1) \\
(\tau,-1,0) \\
(1,0, \tau)\end{array}$ \\
\hline Dodecahedron & $\begin{array}{l}\frac{1}{2}(1,-1,1,1,-1,1) \\
\frac{1}{2}(-1,1,-1,1,1,1) \\
\frac{1}{2}(1,-1,-1,-1,-1,1) \\
\frac{1}{2}(1,1,-1,1,-1,1) \\
\frac{1}{2}(1,1,1,1,-1,-1) \\
\frac{1}{2}(1,1,1,-1,1,-1) \\
\frac{1}{2}(-1,1,-1,1,-1,-1) \\
\frac{1}{2}(-1,-1,1,-1,-1,1) \\
\frac{1}{2}(1,-1,1,1,1,-1) \\
\frac{1}{2}(1,-1,-1,1,-1,-1)\end{array}$ & $\begin{array}{l}(0,1-\tau, \tau) \\
(1,-1,1) \\
(1,1,-1) \\
(1,1,1) \\
(-1,1,1) \\
(\tau-1, \tau, 0) \\
(-\tau, 0,1-\tau) \\
(-\tau, 0,-1+\tau) \\
(-1+\tau,-\tau, 0) \\
(0,1-\tau,-\tau)\end{array}$ \\
\hline Icosidodecahedron & $\begin{array}{l}\frac{1}{2}(1,0,0,-1,0,0) \\
\frac{1}{2}(0,1,0,0,0,1) \\
\frac{1}{2}(0,1,1,0,0,0) \\
\frac{1}{2}(0,0,0,1,0,1) \\
\frac{1}{2}(1,0,0,0,0,1) \\
\frac{1}{2}(1,0,0,0,1,0) \\
\frac{1}{2}(0,0,0,1,1,0) \\
\frac{1}{2}(0,0,0,0,1,1) \\
\frac{1}{2}(0,0,1,1,0,0) \\
\frac{1}{2}(0,0,1,0,-1,0) \\
\frac{1}{2}(1,0,-1,0,0,0) \\
\frac{1}{2}(1,1,0,0,0,0) \\
\frac{1}{2}(0,0,1,0,0,1) \\
\frac{1}{2}(0,1,0,-1,0,0) \\
\frac{1}{2}(0,1,0,0,-1,0)\end{array}$ & $\begin{array}{l}\frac{1}{2}\left(\tau, \tau^{2},-1\right) \\
\frac{1}{2}\left(1, \tau, \tau^{2}\right) \\
\frac{1}{2}\left(-1, \tau, \tau^{2}\right) \\
\frac{1}{2}\left(1,-\tau, \tau^{2}\right) \\
\frac{1}{2}\left(\tau^{2}, \tau\right) \\
(\tau, 0,0) \\
\frac{1}{2}\left(\tau,-\tau^{2}, 1\right) \\
\frac{1}{2}\left(\tau^{2},-1, \tau\right) \\
\frac{1}{2}\left(-1,-\tau, \tau^{2}\right) \\
\frac{1}{2}\left(-\tau^{2}, 1, \tau\right) \\
\frac{1}{2}\left(\tau^{2}, 1,-\tau\right) \\
\frac{1}{2}\left(\tau, \tau^{2}, 1\right) \\
(0,0, \tau) \\
(0, \tau, 0) \\
\frac{1}{2}\left(-\tau, \tau^{2}, 1\right)\end{array}$ \\
\hline
\end{tabular}


Table 2. List of the standard polyhedra and the corresponding translations, relevant to the description of CCMV.

\begin{tabular}{|c|c|c|c|}
\hline shell \# & st. polyhedron & translation & scaling factor \\
\hline 10 & ICO & DOD & $\tau^{2}$ \\
11 & ICO & ICO & $-\tau^{\prime}$ \\
12 & ICO & ICO & 1 \\
13 & ICO & ICO & $\tau$ \\
19 & DOD & ICO & $-2 \tau^{\prime}$ \\
26 & DOD & DOD & $\tau^{2}$ \\
27 & DOD & ICO & $\tau^{\prime 2}$ \\
29 & DOD & ICO & 1 \\
30 & DOD & ICO & $\tau$ \\
44 & IDD & DOD & $\frac{1}{2} \tau$ \\
51 & IDD & ICO & $\frac{1}{2}$ \\
52 & IDD & ICO & $\frac{1}{2} \tau$ \\
53 & IDD & ICO & 1 \\
54 & IDD & ICO & $\frac{1}{2} \tau^{2}$ \\
55 & IDD & ICO & $\tau$ \\
\hline
\end{tabular}

(ii) The icosahedral group commutes with the projection, so that the $6 \mathrm{D}$ preimages of the standard icosahedral polyhedra are, in turn, icosahedral orbits.

(iii) A dilatation by a factor of $\tau$ in 3D corresponds to a symmetry operation of the cubic lattices in $6 \mathrm{D}$.

In particular, we first embed the standard polyhedra into $6 \mathrm{D}$ according to Table 1: the standard icosahedron, icosidedecahedron and dodecahedron can be obtained via projection of the icosahedral orbits of suitable SC, FCC and BCC lattice vectors respectively. Then, since the skeletal double shell structures of the point arrays are combinations of two standard polyhedra at different scalings of $\tau$ (see ${ }^{18}$ and Tables 2,3 ), we create the $6 \mathrm{D}$ counterparts of standard polyhedra rescaled by $\tau$ via the action of the quasidilatation $D$. Finally, the translation vectors $s \in \mathbb{R}^{3}$, along which the double-shell structure is translated to generate the point arrays, also belongs to a rescaled standard polyhedron. Therefore $\boldsymbol{t}=\pi^{-1}(\boldsymbol{s})$ and all its orbit belong to one of the icosahedral lattices.

In this manner, we associate with each point array $S$ a unique set $\Sigma$ of points in either $\mathcal{L}_{S C}, \mathcal{L}_{F C C}$ or $\mathcal{L}_{B C C}$. It is called the lifted viral configuration or lifted point array, and fulfills $\pi(\Sigma)=S$. It follows that $\Sigma$ is the union of icosahedral orbits in $\mathbb{R}^{6}$ and their suitable translates:

$$
\Sigma=\Sigma(\boldsymbol{v}, \boldsymbol{w}, \boldsymbol{t})=\mathcal{I} \boldsymbol{v} \cup \mathcal{I} \boldsymbol{w} \cup(\mathcal{I} \boldsymbol{v}+\mathcal{I} \boldsymbol{t}) \cup(\mathcal{I} \boldsymbol{w}+\mathcal{I} \boldsymbol{t}),
$$

where $\pi \boldsymbol{v}=\boldsymbol{u}, \pi \boldsymbol{w}=\boldsymbol{r}, \pi \boldsymbol{t}=\boldsymbol{s}$.

By construction, all points of a given lifted viral configuration $\Sigma$ are points of some icosahedral lattice, and there exists a unique minimal such lattice that contains a given lifted viral configuration. We say that the lifted point array is embedded into such a lattice, and it can be proved that the minimal lattice containing a given lifted viral configuration $\Sigma$ is icosahedral. 
Table 3. List of generating vectors and minimal lattices for each viral configuration relevant to CCMV

\begin{tabular}{|c|c|c|c|c|}
\hline viral conf. & lattice & $\boldsymbol{v}$ & $\boldsymbol{w}$ & $\boldsymbol{t}$ \\
\hline $10-44$ & SC & $(1,0,0,0,0,0)$ & $(1,1,0,0,1,1)$ & $(1,1,0,0,0,1)$ \\
$26-44$ & BCC & $(1,0,0,1,0,-1)$ & $(1,0,0,0,1,0)$ & $\frac{1}{2}(1,1,1,1,-1,-1)$ \\
$11-27$ & BCC & $\frac{1}{2}(1,-1,1,1,-1,-1)$ & $\frac{1}{2}(1,1,-1,1,1,-1)$ & $\frac{1}{2}(3,-1,1,1,-1,-1)$ \\
$12-27$ & SC & $(1,0,0,0,0,0)$ & $(1,1,0,0,0,1)$ & $(1,0,0,0,0,0)$ \\
$13-27$ & BCC & $(1,1,1,1,1,-2)$ & $\frac{1}{2}(1,1,1,1,-1,-1)$ & $\frac{1}{2}(3,-1,1,1-1,-1)$ \\
$27-51$ & SC & $(0,1,1,0,-1,0)$ & $(0,1,0,0,-1,0)$ & $(1,0,0,0,0,0)$ \\
$27-52$ & SC & $(0,1,0,-1,-1,0)$ & $(0,0,1,0,1,0)$ & $(1,0,0,0,0,0)$ \\
$27-53$ & FCC & $\frac{1}{2}(1,-1,-1,-1,-1,1)$ & $\frac{1}{2}(1,0,1,1,0,-1)$ & $\frac{1}{2}(3,-1,1,1,-1,-1)$ \\
$27-54$ & SC & $(0,1,0,-1,-1,0)$ & $(1,0,1,0,1,-1)$ & $(1,0,0,0,0,0)$ \\
$27-55$ & FCC & $\frac{1}{2}(1,-1,1,1,1,-1)$ & $\frac{1}{2}(1,1,0,2,0,-2)$ & $\frac{1}{2}(3,-1,1,1,-1,-1)$ \\
$27-29$ & BCC & $\frac{1}{2}(1,1,-1,1,-1,1)$ & $\frac{1}{2}(1,3,-1,3,1,-3)$ & $(1,0,0,0,0,0)$ \\
$27-30$ & SC & $(1,1,0,0,0,1)$ & $(1,-1,1,0,0,0)$ & $(1,0,0,0,0,0)$ \\
\hline
\end{tabular}

From the above argument it follows that, since the point arrays can be obtained by projection of a $6 \mathrm{D}$ lattice onto a completely irrational icosahedrally-invariant subspace in $3 \mathrm{D}$, they are subsets of (aperiodic) icosahedral $3 \mathrm{D}$ quasilattices. ${ }^{25}$

\section{Capsid Transitions as 6D Lattice Transitions}

Since we induce transitions in $3 \mathrm{D}$ from lattice transitions in $6 \mathrm{D}$, we first discuss this case in detail.

\section{1. $6 D$ lattice transitions}

For a matrix group $\mathcal{G} \subset G L(6, \mathbb{Z})$, the centralizer $\mathcal{Z}(\mathcal{G}, K)$ of $\mathcal{G}$ in $G L(6, K)$, with $K=\mathbb{Z}, \mathbb{Q}$ or $\mathbb{R}$, is

$$
\mathcal{Z}(\mathcal{G}, K)=\left\{N \in G L(6, K): N^{-1} G N=G, \quad \forall G \in \mathcal{G}\right\} .
$$

Also, we denote by $\mathcal{Z}^{+}(\mathcal{G}, K)$ the connected component of the identity of the set $\{N \in \mathcal{Z}(\mathcal{G}, K): \operatorname{det} N>0\} \subset G L(6, \mathbb{R})$. In short, we define a lattice phase transition as a continuous transformation between two icosahedral lattices $\mathcal{L}\left(B_{0}\right)$ and $\mathcal{L}\left(B_{1}\right)$ along which some symmetry is preserved, described by a common maximal subgroup $\mathcal{G} \subset G L(6, \mathbb{Z})$ of the lattice groups $\Lambda\left(B_{0}\right)$ and $\Lambda\left(B_{1}\right)$. We require that the invariant subspaces $E^{\|}$and $E^{\perp}$ of the icosahedral group $\mathcal{I}$ be invariant under the point group $\mathcal{P}(B(\sigma))$ of the intermediate lattices, so that orbits of $6 \mathrm{D}$ vectors under $\mathcal{P}(B(\sigma))$ project onto orbits or point sets with the same symmetry in $E^{\|}$.

Let $\mathcal{L}_{0}$ and $\mathcal{L}_{1}$ be two icosahedral lattices, and let $\mathcal{G}$ be a maximal subgroup of $\mathcal{I}$. We say that there exists a transition between $\mathcal{L}_{0}$ and $\mathcal{L}_{1}$ with intermediate symmetry $\mathcal{G}$ (and compatible with projection) if there exists a continuous path $B:[0,1] \rightarrow G L(6, \mathbb{R})$ such that

i) $B(0)=B_{0}$ is a basis of $\mathcal{L}_{0}$ and $B(1)=B_{1}$ is a basis of $\mathcal{L}_{1}$; 
ii) the intermediate phases have minimal symmetry $\mathcal{G}$ :

$$
\mathcal{P}(B(\sigma)) \supseteq \mathcal{G}, \quad \sigma \in[0,1] .
$$

We call the linear mapping

$$
T:=B_{1} B_{0}^{-1}: \mathbb{R}^{6} \rightarrow \mathbb{R}^{6},
$$

the transition, while the curve $T(\sigma)=B(\sigma) B_{0}^{-1}$ is referred to as the transition path.

Notice that, in the above definition, we only require that all the intermediate lattices have at least symmetry $\mathcal{G}$ : along the transition path the symmetry of the intermediate lattice may increase for selected values of $\sigma$.

Proposition 3.1. The following statements are equivalent:

i) There exists a transition between $\mathcal{L}_{0}$ and $\mathcal{L}_{1}$ with intermediate symmetry $\mathcal{G}$.

ii) There exist bases $B_{0}$ and $B_{1}$ of $\mathcal{L}_{0}$ and $\mathcal{L}_{1}$ such that

$$
B_{1}=T B_{0}, \quad \text { with } \quad T \in \mathcal{Z}^{+}(\mathcal{G}, \mathbb{Q}) .
$$

iii) There exist a basis $B_{0}$ of $\mathcal{L}_{0}$, and continuous paths

$$
T:[0,1] \rightarrow \mathcal{Z}^{+}(\mathcal{G}, \mathbb{R}),
$$

such that $T(0)=I$ and where $T(1) B_{0}=B_{1}$ is a basis of $\mathcal{L}_{1}$.

The transition paths associated to $T$ are curves

$$
T:[0,1] \rightarrow \mathcal{Z}^{+}(\mathcal{G}, \mathbb{R}),
$$

such that $T(0)=I$ and $T(1)=T$. In this sense, the set $\mathcal{Z}^{+}(\mathcal{G}, \mathbb{R})$ is the parameter space for transitions with intermediate symmetry $\mathcal{G}$. We have computed the centralizers of the maximal subgroups of $\mathcal{I}:{ }^{11}$ for instance, the parameter spaces for tetrahedral, $D_{10}$, and $D_{6}$ transitions are four, six and eight dimensional respectively.

\subsection{Viral transitions}

Consider two viral configurations $S_{0}$ and $S_{1}$, with corresponding lifted viral configurations $\Sigma_{0}$ and $\Sigma_{1}$ in $6 \mathrm{D}$. Let $\Sigma_{0}$ be embedded into $\mathcal{L}_{0}$, and $\Sigma_{1}$ into $\mathcal{L}_{1}$. We define a viral transition between two viral configurations $S_{0}$ and $S_{1}$ in 3D, with intermediate symmetry $\mathcal{G}$ to be a transition obtained via projection $\pi$ from a transition between the lattices $\mathcal{L}_{0}$ and $\mathcal{L}_{1}$ in $6 \mathrm{D}$, such that $B_{0}$ and $B_{1}$ (the bases associated with the transition) are admissible for $\Sigma_{0}$ and $\Sigma_{1}$, i.e.,

$$
\Sigma_{0}=\Sigma\left(B_{0}\right), \quad \Sigma_{1}=\Sigma\left(B_{1}\right),
$$

where an admissible basis for a lifted viral configuration $\Sigma=\Sigma(\boldsymbol{v}, \boldsymbol{w}, \boldsymbol{t})$ is a basis of the minimal icosahedral lattice containing $\Sigma$ such that the $\mathcal{I}$-orbits of the basis vectors and of $\boldsymbol{v}, \boldsymbol{w}, \boldsymbol{t}$ coincide. For $B$ an admissible basis, we write $\Sigma=\Sigma(B)$. 
By Proposition 3.1, given a transition $T$, the possible viral transition paths are curves in $\mathcal{Z}^{+}(\mathcal{G}, \mathbb{R})$ connecting the identity with $T$. To derive from these paths the information about the actual intermediate structure of a viral capsid, let

$$
T(\sigma) \in \mathcal{Z}^{+}(\mathcal{G}, \mathbb{R}), \quad \text { for } \quad \sigma \in[0,1],
$$

be a viral transition path with intermediate symmetry $\mathcal{G}$. Let $\boldsymbol{v}_{0}, \boldsymbol{w}_{0}$ and $\boldsymbol{t}_{0}$ be three vectors of the basis $B_{0}$ such that $\Sigma_{0}=\Sigma\left(\boldsymbol{v}_{0}, \boldsymbol{w}_{0}, \boldsymbol{t}_{0}\right)$, For $\sigma \in(0,1)$, define

$$
\boldsymbol{v}(\sigma)=T(\sigma) \boldsymbol{v}_{0}, \quad \boldsymbol{w}(\sigma)=T(\sigma) \boldsymbol{w}_{0}, \quad \boldsymbol{t}(\sigma)=T(\sigma) \boldsymbol{t}_{0} .
$$

By definition, $\boldsymbol{v}(\sigma), \boldsymbol{w}(\sigma)$ and $\boldsymbol{t}(\sigma)$ are vectors of the basis $B(\sigma)=T(\sigma) B_{0}$ of the intermediate lattice. We associate with the transition path $T(\sigma)$, for any $\sigma \in(0,1)$, a lifted viral configuration $\Sigma(\sigma)$ defined as

$$
\Sigma(\sigma)=\mathcal{G} \boldsymbol{v}(\sigma) \cup \mathcal{G} \boldsymbol{w}(\sigma) \cup(\mathcal{G} \boldsymbol{v}(\sigma)+\mathcal{G} \boldsymbol{t}(\sigma)) \cup(\mathcal{G} \boldsymbol{w}(\sigma)+\mathcal{G} \boldsymbol{t}(\sigma)) \subset \mathcal{L}(B(\sigma)) .
$$

The resulting point array, when projected to $\mathbb{R}^{3}$ via $\pi$, yields a family of nonicosahedral point sets $S(\sigma)$ parametrized by $\sigma$, with constant $\mathcal{G}$-symmetry, that encode structural boundary conditions on the intermediate viral configurations.

\section{Application to CCMV}

We apply the procedure outlined in the previous sections to CCMV capsid transitions during maturation. An analysis of the CCMV capsid structure via the algorithm in Keef et al. ${ }^{15}$ shows that the pre-transition geometry of the CCMV capsid is given by one of the two following viral configurations (see Section 2 and Tables 2, 3 for the notations used to label the point-arrays):

$$
S_{0} \in\{10-44,26-44\}
$$

while the swollen form of CCMV is best approximated by one of the ten following viral configurations:

$$
S_{1} \in\{11-27,12-27,13-27,27-29,27-30,27-51,27-52,27-53,27-54,27-55\} .
$$

We have determined all possible Bain transitions with capsid configurations given by a point array $S_{0}$ in (4), and post-transition configuration given by an array $S_{1}$ in (5). The results are as follows:

i) There exist no Bain transitions with either $A_{4}$ or $D_{10}$ symmetry between any of the initial and final configurations.

ii) There exist four Bain transitions with $D_{6}$ symmetry, mapping the initial configuration 10-44 into one of the final configurations 27-52, 11-27 and 12-27.

It is not possible to fully determine the transition path explicitly without further insights into the energetic contributions to these transitions. However, our analysis provides fundamental insights into the likely symmetry of the transition path. Our results for CCMV imply that the configurations of the CCMV capsid during the transition will not be icosahedral, unlike the start and end structure, and will at most 
have $D_{6}$ symmetry. $D_{6}$, a subgroup of the icosahedral group, has a representation as the dihedral group of a triangular prism, with one distinguished three-fold axis. Assuming that the particle will maximise symmetry throughout the transition, this implies that a 3 -fold axis will play a crucial role during the structural transition. This result provides important insights into the transition process because it relates to a phenomenon observed for transition events in viruses: Structural transitions seem to start at a given symmetry axis of a spherical particle, e.g. a 5-fold axis, and then propagate over the surface of the capsid like a circular wave until the entire particle has undergone the transition. ${ }^{28}$ This implies that intermediate configurations are presumably preserving one of the symmetry axis, and our method can be used to determine a priori which symmetry axis this is most likely to be.

\section{Conclusion}

Due to the occurence of symmetry in the organisation of viruses, their structures can be analysed via group theory. We have shown here that affine extensions of the icosahedral group provide new insights into the structural interdependence of all components of a virus, and that such results can be used to understand virus function. We have focused here on the structural transitions important for infection. However, symmetry impacts also on other stages of the viral life-cycle, such as the assembly of the protein containers. Insights from Keef et al. have also been used in this context, $5,10,14,21,30$ and have been instrumental in elucidating the cooperative roles of the viral genome in RNA virus assembly. This shows that the fundamental geometric principle in virology encoded by the library of point arrays has wide applications in virology.

\section{Acknowledgments}

RT would like to thank the Leverhulme Trust for funding of her research team via a Research Leadership Award. GI acknowledges funding by the Marie Curie Project MATVIR. Both RT and GI would like to thank the University of Putra Malaysia, INSPEM, for their kind hospitality.

\section{References}

1. P. Alippi, P. M. Marcus and M. Scheffler, Phys. Rev. Lett. 78/20, 3892-3895 (1997).

2. E. C. Bain, Trans. AIME, 70, 25-35 (1924).

3. K. Bhattacharya, S. Conti, G. Zanzotto and J. Zimmer, Nature 428, 55-59 (2004).

4. L. L. Boyer, Acta Cryst. A45, fc29-fc32 (1989).

5. D. H. J. Bunka, S. W. Lane, C. L. Lane, E. C. Dykeman, R. Ford, A. Barker, R. Twarock, S. E. V. Phillips and P. G. Stockley. Evidence for Dispersed, Degenerate RNA Packaging Signals in the Genome of Satellite Necrosis Virus: Implications for the Assembly of a T=1 Capsid, to appear in J. Mol. Biol. (2011).

6. C. Capillas, J. M. Perez-Mato and M. I. Aroyo, J. Phys.: Condens. Matter 19 27-5203 (2007). 
7. D. L. D. Caspar and A. Klug A., Physical principles in the construction of regular viruses in Cold Spring Harbor Symp. 27:1-24, (1962).

8. J. W. Christian, The theory of transformations in metals and alloys (Pergamon Press, Oxford, 2002).

9. F. H. Crick and J. D. Watson, Nature 177473 (1956).

10. E. C. Dykeman, N. Grayson, N. A. Ranson, P. G. Stockley and R. Twarock, J. Mol. Biol. 408399 (2011).

11. G. Indelicato, P. Cermelli, D. G. Salthouse, S. Racca, G. Zanzotto and R. Twarock, J. Math. Biol. DOI: 10.1007/s00285-011-0425-5 (2011).

12. A. Katz, Some local properties of the 3D Penrose tilings, in Introduction to the Mathematics of Quasicrystals, M. V. Jaric ed. (Academic Press, 1989), p. 147.

13. E. Kaxiras and L. L. Boyer, Modelling Simul. Mater. Sci. Eng. 1, 91-100 (1992).

14. T. Keef, C. Micheletti and R. Twarock, J. Theor. Biol. 242713 (2006).

15. T. Keef, J. Wardman, N. A. Ranson, P. G. Stockley and R. Twarock, Viruses measure up to mathematical prediction - 3D Geometry imposes fundamental constraints on the structures of simple viruses (submitted).

16. T. Keef and R. Twarock, Beyond Quasi-Equivalence: New Insights Into Viral Architecture via Affine Extended Symmetry Groups in Emerging Topics in Physical Virology (Imperial College Press, London, 2010), p. 59-83

17. T. Keef, R. Twarock and K. M. Elsawy J. Theor. Biol. 253 (2008).

18. T. Keef and R. Twarock, J. Math. Biol. 59, 287-313 (2009).

19. L. S. Levitov and J. Rhyner, J. Physique, 49, 1835-1849 (1988).

20. M. J. Mehl, A. Aguayo and L. L. Boyer, Phys. Rev. B 70, 014105 (2004).

21. V. L. Morton et al., J. Mol. Biol. 401, 298 (2010).

22. M. Pitteri and G. Zanzotto, Acta Cryst. A54, 359-373 (1998).

23. M. Pitteri and G. Zanzotto, Continuum models for phase transitions and twinning in crystals (CRC/Chapman and Hall, London, 2002).

24. I. K. Robinson and S. C. Harrison, Nature 297563 (1982).

25. M. Senechal, Quasicrystals and geometry (Cambridge University Press, Cambridge, 1995).

26. M. B. Sherman, H. R. Guenther, F. Tama, T. L. Sit, C. L. Brooks, A. M. Mikhailov, E. V. Orlova, T. S. Baker and S. A. Lommel, J. Virol. 80/21 10395 (2006).

27. J. A. Speir, S. Munshi, G. Wang, T. S. Baker and J. E. Johnson, Structure 3 63, (1995).

28. Steven A., Private communication, (2010).

29. P. Toledano and V. Dmitriev, Reconstructive phase transitions (World Scientific Publishing Company, 1996).

30. K. Toropova, G. Basnak, R. Twarock, P.G. Stockley and N. A. Ranson, J. Mol. Biol. 375824 (2008).

31. R. Twarock, J. Theor. Biol. 226, 477 (2004).

32. R. Twarock and T. Keef, Microbiology Today 37 24-27 (2010).

33. http://viperdb.scripps.edu/

34. J. Wardman, Private communication, (2010).

35. C. M. Wayman, Introduction to the crystallography of martensite transformations (Macmillan, New York, 1964). 